\title{
Quasi-Soliton Pulse-Train Propagation in Dispersion-Managed Silicon Rib Waveguides
}

\author{
Odysseas Tsilipakos, Student Member, IEEE, Dimitrios C. Zografopoulos, Member, IEEE, and \\ Emmanouil E. Kriezis, Senior Member, IEEE
}

\begin{abstract}
Dispersion-managed soliton-like pulse-train propagation in silicon rib waveguides is theoretically investigated by means of the nonlinear Schrödinger equation. A vectorial formulation is employed taking rigorously into account all relevant phenomena. For the purpose of minimizing pulse broadening, the waveguide width is precisely engineered (tapered) along the propagation direction by demanding that the soliton formation condition is locally satisfied. For typical carrier lifetimes (3 ns), bit rates up to $160 \mathrm{Gbit} / \mathrm{s}$ can be transmitted with negligible pulse broadening and less than $3 \mathrm{~dB}$ of free-carrier-induced loss. The application of a carrier-sweep bias allows for the extension of the transmission rate beyond $1.28 \mathrm{Tbit} / \mathrm{s}$, matching current trends in silicon-photonic optical time-division multiplexing systems.
\end{abstract}

Index Terms-Nonlinear pulse propagation, temporal solitons, dispersion management, silicon rib waveguides.

\section{INTRODUCTION}

$\mathbf{N}$ ONLINEAR phenomena in silicon photonic waveguides (SPWs) have been under intense investigation in recent years, due to their potential for functional, low-cost, monolithically-integrated photonic circuits, capable of ultrafast optical signal processing [1], [2]. In particular, the capability for extensive dispersion engineering in compact SPWs [3], [4] has directed considerable interest to the dynamics of femtosecond solitonic pulses [5], [6]. Soliton-like propagation provides a dispersion-free format for chip-scale applications, e.g., high bit-rate optical interconnects based on optical timedivision multiplexing (OTDM) [7]. However, the formation of pure solitons in SPWs is hindered (besides linear losses) by the presence of nonlinear losses, namely, two-photon (TPA) and free-carrier absorption (FCA). Despite these extra loss mechanisms, we have recently demonstrated the possibility for quasi-soliton propagation of a single pulse in properlypatterned wire SPWs, in a preliminary feasibility study where various physical aspects were qualitatively assessed [8].

In this Letter, we comprehensively study fs-pulse propagation in dispersion-managed SPWs, focusing this time on rib waveguides since they can allow for carrier sweeping.

Manuscript received December 18, 2012. This work was supported in part by the European Union (European Social Fund, ESF) and Greek national funds through the Operational Program "Education and Lifelong Learning" of the National Strategic Reference Framework (NSRF). Research Funding Program THALES "Reinforcement of the interdisciplinary and/or inter-institutional research and innovation" (Project ANEMOS).

O. Tsilipakos and E. E. Kriezis are with the Department of Electrical and Computer Engineering, Aristotle University of Thessaloniki, Thessaloniki GR54124, Greece. (e-mail: otsilipa@ auth.gr; mkriezis@ auth.gr)

D. C. Zografopoulos is with the Consiglio Nazionale delle Ricerche, Istituto per la Microelettronica e Microsistemi (CNR-IMM), Roma 00133, Italy (email: dimitrios.zografopoulos@artov.imm.cnr.it).
Both single-pulse and, more importantly, pulse-train cases are thoroughly examined, by employing a vectorial formulation taking into account all relevant phenomena. Specifically, we include dispersion to all orders, carrier generation and accumulation, the contribution of both transverse and axial mode components, third-order susceptibility anisotropy, and selfsteepening. We investigate into the maximum achievable pulse repetition rate, with an eye on OTDM applications in integrated silicon photonic circuits. We show that by employing a carrier-sweep bias, bit rates beyond $1.28 \mathrm{Tbit} / \mathrm{s}$ are possible with virtually no pulse distortion.

\section{THEORETICAL FRAMEWORK}

Pulse propagation dynamics are modeled with the generalized nonlinear Schrödinger equation (NLSE) [9]

$$
\begin{aligned}
\frac{\partial A}{\partial z} & =\sum_{m=2}^{\infty} i^{m+1} \frac{\beta_{m}}{m !} \frac{\partial^{m} A}{\partial t^{m}}+i \gamma\left(1+i \tau_{\mathrm{s}} \frac{\partial}{\partial t}\right)|A|^{2} A+ \\
& +B\left(i k_{0} \Delta n^{\mathrm{fc}}-\frac{\Delta \alpha^{\mathrm{fc}}}{2}\right) A-\frac{\alpha_{l}}{2} A,
\end{aligned}
$$

where $A$ is the pulse envelope (such that $|A|^{2}$ is the mode power) and $\alpha_{l}$ the linear loss coefficient, equal to $2 \mathrm{~dB} / \mathrm{cm}$ [10]. In (1) we have made use of the retarded frame transformation $\left(T=t-\beta_{1} z\right)$, retaining, however, the variable $t$ for describing the retarded time $T$.

The infinite sum describes frequency dispersion, with $\beta_{m}=\partial^{m} \beta / \partial \omega^{m}$ denoting the $m$-th order dispersion parameter. Instead of truncating the sum, dispersion is included to all orders by replacing it in the Fourier domain with $i\left[\beta(\omega)-\beta\left(\omega_{0}\right)-\beta_{1}\left(\omega_{0}\right)\left(\omega-\omega_{0}\right)\right] \tilde{A}$ [4], [11]. $\beta(\omega)$ is calculated with the finite-element method, taking into account the material dispersion of $\mathrm{Si}$ and $\mathrm{SiO}_{2}$ [12].

The effect of self-phase modulation (SPM) and TPA is introduced via the complex nonlinear coefficient $\gamma$ [9]

$$
\gamma=\frac{3 \omega \varepsilon_{0}}{4} \sum_{\mu \alpha \beta \gamma}^{x, y, z} \frac{\iint \chi_{\mu \alpha \beta \gamma}^{(3)} e_{\mu}^{*} e_{\alpha} e_{\beta}^{*} e_{\gamma} \mathrm{d} x \mathrm{~d} y}{\left[\iint \mathbf{e} \times \mathbf{h}^{*} \cdot \hat{\mathbf{z}} \mathrm{d} x \mathrm{~d} y\right]^{2}},
$$

where we have assumed [13]: $n_{2, \mathrm{Si}}=6 \times 10^{-18} \mathrm{~m}^{2} / \mathrm{W}$, $n_{2, \mathrm{SiO}_{2}}=2.6 \times 10^{-20} \mathrm{~m}^{2} / \mathrm{W}$, and $\beta_{\mathrm{TPA}}=5 \times 10^{-12} \mathrm{~m} / \mathrm{W}$. This definition accounts for waveguide cross-section inhomogeneity (i.e., linear and nonlinear indices are functions of the cross-sectional coordinates), the hybrid nature of the supported mode (nonzero axial field component), and $\mathrm{Si} \chi^{(3)}$ anisotropy. The waveguide is assumed along the $[\overline{1} \overline{1} 0]$ direction and thus the ratio of the two independent $\chi^{(3)}$ components equals 
$\rho \equiv 3 \chi_{1122}^{(3)} / \chi_{1111}^{(3)}=1.27$ [9], [14]. Self steepening (SS) is described by the shock time $\tau_{s}=\left.(\partial \ln \gamma / \partial \omega)\right|_{\omega_{0}}$.

The term involving the overlap factor $B$, defined as [9]

$$
B=\frac{n_{\mathrm{Si}}}{\eta_{0}} \frac{\iint_{\mathrm{Si}}|\mathbf{e}|^{2} \mathrm{~d} x \mathrm{~d} y}{\iint \mathbf{e} \times \mathbf{h}^{*} \cdot \hat{\mathbf{z}} \mathrm{d} x \mathrm{~d} y},
$$

describes free-carrier effects, namely, dispersion (FCD) and absorption. In (3), $\eta_{0}$ denotes the intrinsic impedance of free space. At the working wavelength of $1.55 \mu \mathrm{m}: \Delta \alpha^{\mathrm{fc}}=\sigma_{\alpha} N_{\mathrm{c}}$, $\Delta n^{\text {fc }}=-\sigma_{n}^{e} N_{\mathrm{c}}-\left(\sigma_{n}^{h} N_{\mathrm{c}}\right)^{0.8}$, with $\sigma_{\alpha}=1.45 \times 10^{-17} \mathrm{~cm}^{2}$, $\sigma_{n}^{e}=8.8 \times 10^{-22} \mathrm{~cm}^{3}$, and $\sigma_{n}^{h}=4.6 \times 10^{-22} \mathrm{~cm}^{3}$ [9]. The TPA-induced FC density $N_{\mathrm{c}}$ is specified by solving the following rate equation [9]

$$
\frac{\partial N_{\mathrm{c}}(z, t)}{\partial t}=\frac{\operatorname{Im} \gamma}{\hbar \omega_{0} A_{0}}|A(z, t)|^{4}-\frac{N_{\mathrm{c}}(z, t)}{\tau_{\mathrm{c}}},
$$

where $A_{0}$ is the waveguide core area and $\tau_{\mathrm{c}}$ the carrier lifetime that in the absence of carrier sweeping is set to 3 ns [10], [11].

We note that the effect of intrapulse Raman scattering is not included in (1), since the pulse bandwidth is lower than the 15.6 THz Raman shift for silicon [5]. Equivalent formulations with the one adopted have been described in [15] and, with the exception of TPA and the resulting FC effects, [16].

The numerical simulations are carried out with the symmetrized version of the split-step Fourier method [17]. The FC density is calculated at the middle of each propagation step. For the pulse-train case, contrary to previous approaches [13], (4) is transformed to the frequency domain and solved with the help of the FFT. This means that automatically a steady-state condition for the FC density is assumed, which would otherwise be reached after $\sim 5 \tau_{\mathrm{c}}$ [13]. Given that this way periodicity is inherently satisfied, only a single period of the pulse-train needs be considered, as done for (1), saving computational resources.

\section{Silicon Rib WaVEguide}

The SPW under study is a $340-\mathrm{nm}$-thick rib waveguide [Fig. 1(a)], operating on the fundamental TE-like mode. The waveguide width $w$ is considered a design parameter and the slab thickness is $40 \mathrm{~nm}$. Similar rib waveguides have been shown to allow for record-fast FC sweeping via reversebiased $p-i-n$ structures [10]. Figure 1(b) depicts group-velocity (GVD) and third-order (TOD) dispersion parameters as functions of the waveguide width in the range $320-420 \mathrm{~nm}$. For the most part, dispersion remains anomalous (a prerequisite for soliton formation) exhibiting a maximum at $400 \mathrm{~nm}$ and monotonically decreasing for smaller widths. In the same way, Fig. 1(c) depicts real and imaginary parts of the complex nonlinear coefficient $\gamma$. The corresponding real part of $\tau_{\mathrm{s}}$ is found to drop monotonically in the said interval from 2.5 to $1.5 \mathrm{fs}$ [Fig. 1(d)], whereas its imaginary part is almost constant and equal to -0.08 fs. Finally, the index overlap factor $B$ exhibits a rather weak variation between 1.172 and 1.193, Fig. 1(d). In order to minimize the detrimental effect of TOD (it distorts the pulse shape thus impeding quasi-soliton formation) [8], the waveguide width is initially set to $w_{0}=390 \mathrm{~nm}$, for which $\beta_{3}$ is approximately zero $\left(\beta_{3}=5.7 \times 10^{-4} \mathrm{ps}^{3} / \mathrm{m}\right)$, Fig. 1(b). The remaining width-sensitive parameters acquire
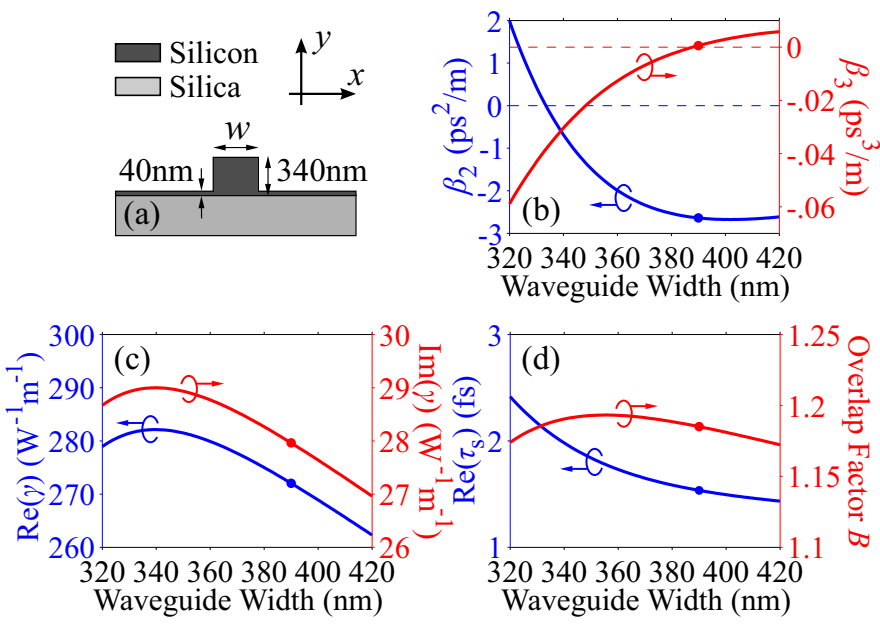

Fig. 1. (a) Silicon rib waveguide under study. (b) GVD and TOD parameters as functions of waveguide width. (c) Real and imaginary parts of complex nonlinear parameter $\gamma$ as functions of waveguide width. (d) Real part of shock time $\tau_{\mathrm{s}}$ and index overlap factor $B$ as functions of waveguide width. The parameter values for a width of $390 \mathrm{~nm}$ (the starting width of the proposed tapered waveguide) are marked with dots. Note that $\beta_{3}$ is approximately zero.

the following values [Fig. 1(b)-(d)]: $\beta_{2}=-2.64 \mathrm{ps}^{2} / \mathrm{m}$, $\gamma=272+i 28 \mathrm{~W}^{-1} \mathrm{~m}^{-1}, \tau_{\mathrm{s}}=1.5-i 0.08$ fs and $B=1.185$.

\section{Single Pulse Propagation}

First, the case of a single pulse is considered. The amplitude profile is given by $A(0, t)=\sqrt{P_{0}} \operatorname{sech}\left(t / T_{0}\right)$ with $T_{0}=100 \mathrm{fs}$. The input power is determined from the soliton formation condition (SFC) $L_{\mathrm{NL}, 0}=L_{\mathrm{D}, 0}$ and equals $\sim 1 \mathrm{~W}$; a power level readily supplied by high-power erbium-doped fiber amplifiers. $L_{\mathrm{NL}, 0}=\left(\operatorname{Re}\left\{\gamma\left(w_{0}\right)\right\} P_{0}\right)^{-1}$ is the nonlinear and $L_{\mathrm{D}, 0}=T_{0}^{2} /\left|\beta_{2}\left(w_{0}\right)\right|$ the dispersion length. After each propagation step $i \Delta z$, the width $w^{i+1}$ that locally satisfies the SFC $L_{\mathrm{D}, z}=L_{\mathrm{NL}, z}$ is calculated, yielding the profile $w(z)$ that minimizes RMS pulse broadening [8]. For this purpose, the width-sensitive parameters $\left(\beta_{i}, \gamma, \tau_{\mathrm{s}}, B\right)$ have to be functionalized with respect to $w$. We finally note that waveguide width engineering has been utilized to enhance diverse nonlinear processes, such as four wave mixing in [18].

Figure 2(a) depicts the output pulse at the end of a properlytapered $L=10 L_{\mathrm{D}, 0}=3.8$-cm-long waveguide. The input pulse is also included for comparison purposes (dashed line). Note the different scales of respective $y$-axes. The insertion loss of $10 \mathrm{~dB}$ is due to propagation loss $(7.6 \mathrm{~dB})$ and TPA. Importantly, pulse broadening is negligible, indicating quasi-soliton propagation. This can be verified by observing Fig. 2(b), where the corresponding width profile and evolution of RMS pulse broadening are plotted. The latter remains lower than $6 \%$ along the waveguide, verifying quasi-soliton propagation for the single-pulse case. The dashed curve in Fig. 2(b) demonstrates the extreme broadening that would have been experienced by the pulse, had we kept the waveguide width constant at $390 \mathrm{~nm}$. Specifically, at the waveguide end the pulse would have broadened by $250 \%$ (not shown).

It is worth noting that the required tapering is very smooth and thus not expected to induce additional losses. Specifically, the maximum tapering rate $\mathrm{d} w / \mathrm{d} z$, observed at the waveguide 

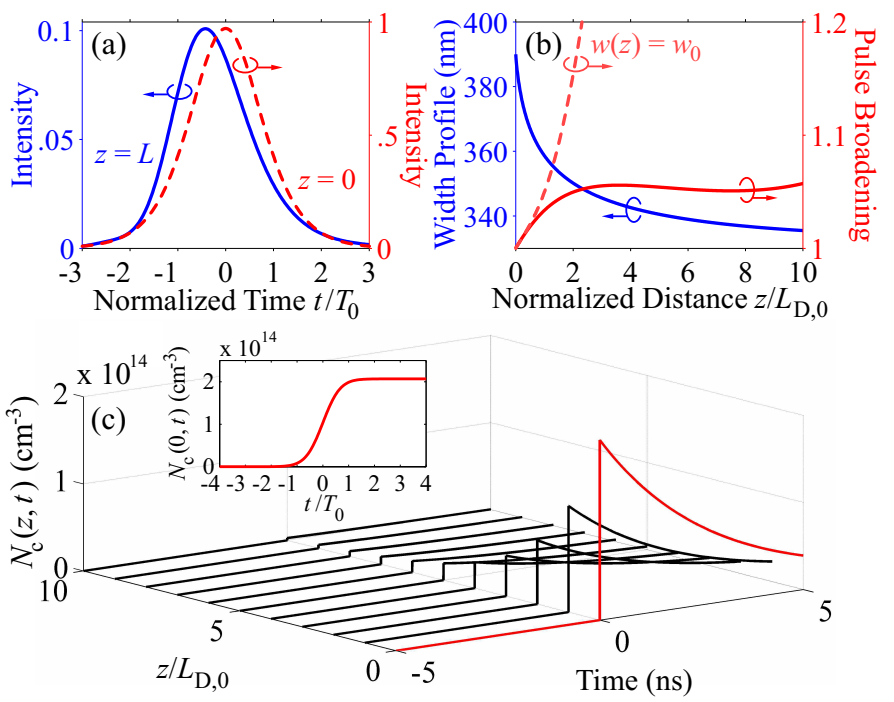

Fig. 2. (a) Output pulse at the end of a properly-tapered 3.8-cm-long waveguide. The input pulse is included with a dashed line. Note the different scales of respective $y$-axes. (b) Corresponding width profile $w(z)$ and RMS pulse broadening along the waveguide. Pulse broadening for a waveguide with constant width, $w(z)=w_{0}$, is included with a dashed line. (c) FC density evolution along the waveguide. Inset: Detail at $z=0$ illustrating how the maximum density is reached after the entire pulse has passed. This density value is retained before slowly dropping due to FC recombination.

input, is only $\sim 30 \mathrm{~nm} / \mathrm{mm}$, corresponding to a tapering angle orders-of-magnitude smaller than those typical of Si-photonic adiabatic linear horizontal tapers [19]. As an additional practical concern, we have checked that systematic fabrication errors offsetting the optimum width profile by a constant value $\leq \pm 2 \mathrm{~nm}$ can be fully compensated; by properly adjusting the input power we can match the performance of the ideal taper.

The temporal asymmetry of the output pulse in Fig. 2(a) originates almost exclusively from the progressively increasing values of $\left|\beta_{3}\right|$, as the waveguide width decreases. Oscillations (a trademark of $\beta_{3}$-induced pulse asymmetry) do not develop near the leading edge of the pulse since the waveguide length does not exceed the TOD length $L_{\mathrm{D}}^{\prime}=T_{0}^{3} /\left|\beta_{3}\right|$ [17]. More specifically, the mean value of $\left|\beta_{3}\right|$ experienced by the pulse along the waveguide is $\sim 2.6 \times 10^{-2} \mathrm{ps}^{3} / \mathrm{m}$ [cf. Fig. 1(b)], resulting in a mean TOD length of $\sim 3.8 \mathrm{~cm}$, equal to the waveguide length. For unit values of the $L / L_{D}^{\prime}$ ratio (in the absence of SPM-induced spectral broadening), the effect of TOD is to simply shift the pulse; oscillations appear for higher ratio values [17]. The impact of SS is marginal, as the shock times involved ( $\sim 2 \mathrm{fs})$ are not sufficient to induce significant pulse peak shifting [14]. The FC effects were found negligible as well, owing to the low energy of the fs pulse $(\sim 200 \mathrm{fJ}$ at waveguide input), resulting in very low FC densities. More specifically, as demonstrated in Fig. 2(c) where FC density evolution along the waveguide is presented, the maximum density at the waveguide input is $2 \times 10^{14} \mathrm{~cm}^{-3}$. Thus, FCA is roughly two orders of magnitude lower than propagation loss and FCD $\sim 25$ times smaller than SPM-induced index modulation.
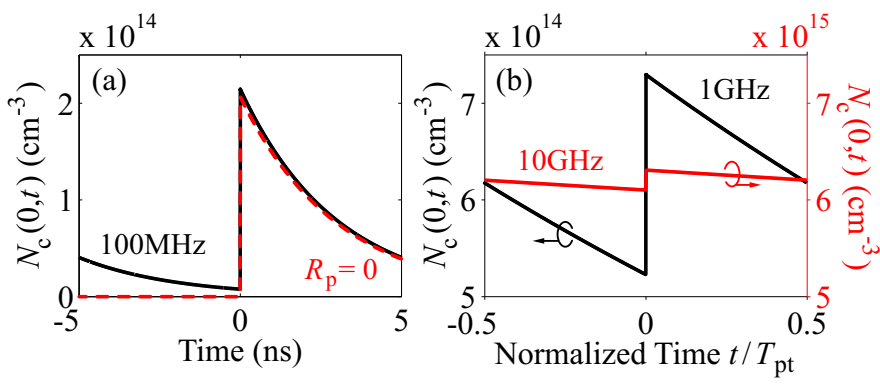

Fig. 3. FC density at waveguide input for several pulse repetition rates. (a) $R_{\mathrm{p}}=100 \mathrm{MHz}$. The single-pulse case $\left(R_{\mathrm{p}}=0\right)$ is included with a dashed line for comparison purposes. (b) $R_{\mathrm{p}}=1,10 \mathrm{GHz}$. Note that for $R_{\mathrm{p}}=10 \mathrm{GHz}$ the FC density is almost constant across the pulse-train period.
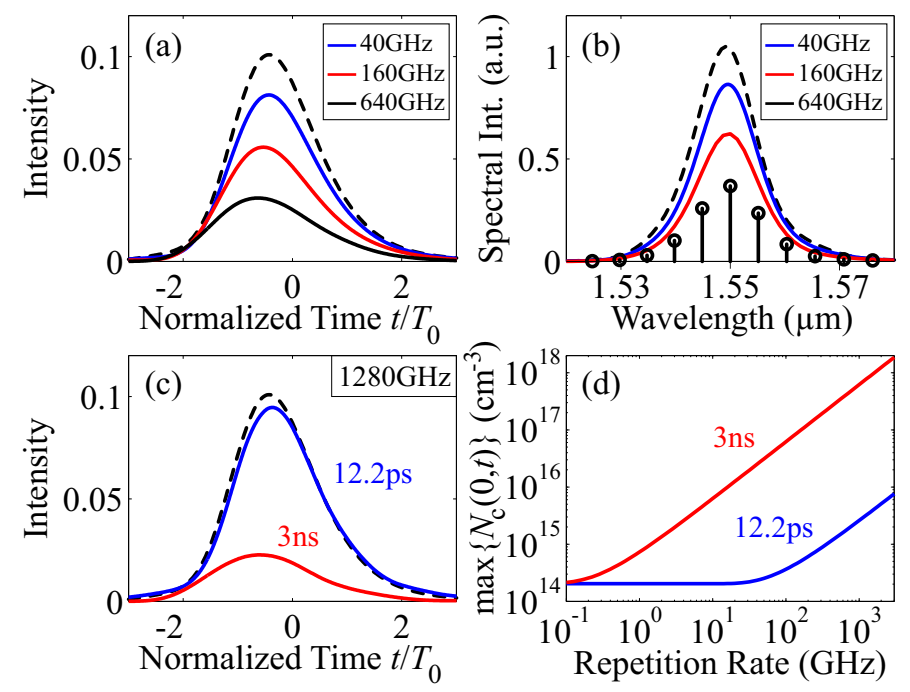

Fig. 4. Effect of repetition rate on output pulse: (a) Temporal and (b) spectral domains. The single-pulse case is included with a dashed line. The stem plot denotes the discrete nature of the pulse-train spectrum. Carrier lifetime is 3 ns. (c) Output pulse for the two carrier lifetime cases (3 and $12.2 \mathrm{~ns}$ ) when $R_{\mathrm{p}}=1280 \mathrm{GHz}$. (d) Maximum carrier density at waveguide input $(z=0)$ as a function of repetition rate for the two carrier lifetime cases.

\section{Pulse Train Propagation}

Although the influence of FCs in the single pulse case is negligible, for high bit rates it is naturally expected that FC accumulation from successive pulses will impact pulsetrain propagation. This FC accumulation is demonstrated in Fig. 3, which depicts FC density at the waveguide input for several pulse repetition rates $R_{\mathrm{p}}$. At low rates up to $100 \mathrm{MHz}$ [Fig. 3(a)], FCs have enough time to recombine and the maximum density is almost identical with that of the single pulse (dashed line). This comes as no surprise since the pulse-train period, $T_{\mathrm{pt}}=1 / R_{\mathrm{p}}=10 \mathrm{~ns}$, is longer than the carrier lifetime $(3 \mathrm{~ns})$. On the other hand, at higher rates the maximum FC density increases [Fig. 3(b)]. Progressively, carriers generated from further-away pulses are contributing to the result and the density variation across the pulse-train period is reduced. For example, $R_{\mathrm{p}}=10 \mathrm{GHz}$ results in an almost constant across the pulse-train period $\mathrm{FC}$ density, equal to $\sim 6.2 \times 10^{15} \mathrm{~cm}^{-3}$. However, such density values are still rather low and do not result in significant FCA or FCD.

FC effects start impacting pulse-train propagation for higher 
repetition rates. This is demonstrated in Fig. 4(a),(b), compiling output pulses and pulse spectra for $R_{\mathrm{p}}=40,160$ and $640 \mathrm{GHz}$. The corresponding (constant w.r.t. time) carrier densities at the waveguide input are $2.5 \times 10^{16}, 10^{17}$ and $4 \times 10^{17} \mathrm{~cm}^{-3}$, respectively. Each scenario corresponds to an optimal width profile calculated with the general SFC, taking into account all loss mechanisms, linear and nonlinear ones. The derived profiles are qualitatively very similar with that of the single-pulse case [Fig. 2(b)]; albeit, as the bit rate (and thus FCA) increases they become steeper near the waveguide input where FCA is strongest. Note, however, that the tapering remains very smooth; even in the $640-\mathrm{GHz}$ case, the maximum tapering rate does not exceed $200 \mathrm{~nm} / \mathrm{mm}$.

Observing Fig. 4(a) one can confirm that FCA induces additional losses, which become more significant as the bit rate increases. However, it does not induce pulse, and consequently pulse-spectrum, asymmetry [Fig. 4(a),(b)]. This is because the FC density profile $N_{\mathrm{c}}(t)$ is constant across the pulsetrain period, as already indicated by Fig. 3(b). In the same way, FCD acting in a constant fashion does not broaden or blue-shift the pulse spectrum [13]. Since FC effects do not distort the pulse shape, the SFC can still successfully hinder pulse broadening. Specifically, even in the $640-\mathrm{GHz}$ case, RMS broadening does not exceed $11 \%$, verifying quasisoliton propagation for the pulse-train as well. Consequently, the maximum achievable rate is loss-limited, an upper limit being $160 \mathrm{GHz}$ leading to $\sim 3 \mathrm{~dB}$ of free-carrier-induced loss [Fig. 4(a)]. The slight increase in broadening with repetition rate can be explained as follows: As already mentioned, as FCA becomes more significant due to carrier accumulation, the SFC dictates steeper tapering, especially near the waveguide input where FCA is strongest. Therefore, larger values of $\left|\beta_{3}\right|$ are experienced by the pulse, preventing the SFC to hinder broadening as effectively. These larger $\left|\beta_{3}\right|$ values are also reflected on the induced pulse asymmetry [Fig. 4(a)].

Pushing the transmission rate to even higher numbers would demand for a carrier sweeping mechanism, such as reverse biasing of a $p-i-n$ diode. Then, carrier lifetime can drop down to values as low as $12.2 \mathrm{ps}$ [10], enabling transmission rates beyond $1.28 \mathrm{Tbit} / \mathrm{s}$. This is demonstrated in Fig. 4(c), depicting output pulses for $R_{\mathrm{p}}=1280 \mathrm{GHz}$. In the event of carrier sweeping, the pulse is almost identical with the single-pulse case. This is justified by the results of Fig. 4(d), where the maximum (w.r.t. time) carrier density at the waveguide input is plotted for the two lifetime scenarios. For $\tau_{\mathrm{c}}=12.2 \mathrm{ps}$, the maximum current density at $1280 \mathrm{GHz}$ acquires the value of $\sim 3.3 \times 10^{15} \mathrm{~cm}^{-3}$, which is not high enough to induce significant FCA losses. Note that the threshold value after which maximum density begins to rise corresponds to approximately $\left(5 \tau_{\mathrm{c}}\right)^{-1} \sim 16.4 \mathrm{GHz}$.

\section{CONCLUSION}

In summary, the quasi-soliton propagation of 100-fs-longpulse trains in dispersion-decreasing silicon rib waveguides has been rigorously investigated. We have demonstrated that for typical carrier recombination times (3 ns) transmission rates up to $160 \mathrm{Gbit} / \mathrm{s}$ are possible with moderate FCAinduced losses $(<3 \mathrm{~dB})$. The application of carrier sweeping allows for the extension of the transmission rate beyond $1.28 \mathrm{Tbit} / \mathrm{s}$. As a result, the proposed quasi-solitons could find use in silicon photonics-based OTDM systems, providing an alternative route towards on-chip high-bit-rate transmission to dense wavelength-division multiplexing [20].

\section{REFERENCES}

[1] J. Leuthold, C. Koos, and W. Freude, "Nonlinear silicon photonics," Nat. Photon., vol. 4, no. 8, pp. 535-544, Aug. 2010.

[2] Q. Lin, O. J. Painter, and G. P. Agrawal, "Nonlinear optical phenomena in silicon waveguides: modeling and applications," Opt. Express, vol. 15, no. 25, pp. 16604-16644, Dec. 2007.

[3] X. Chen, N. C. Panoiu, I. Hsieh, J. I. Dadap, and R. M. Osgood, Jr., "Third-order dispersion and ultrafast-pulse propagation in silicon wire waveguides," IEEE Photon. Technol. Lett., vol. 18, no. 24, pp. 26172619, Dec. 2006

[4] R. M. Osgood, Jr., N. C. Panoiu, J. I. Dadap, X. Liu, X. Chen, I. Hsieh, E. Dulkeith, W. M. J. Green, and Y. A. Vlasov, "Engineering nonlinearities in nanoscale optical systems: physics and applications in dispersion-engineered silicon nanophotonic wires," Adv. Opt. Photon., vol. 1, no. 1, pp. 162-235, Jan. 2009.

[5] L. Yin, Q. Lin, and G. P. Agrawal, "Dispersion tailoring and soliton propagation in silicon waveguides," Opt. Lett., vol. 31, no. 9, pp. 12951297, May 2006.

[6] J. Zhang, Q. Lin, G. Piredda, R. W. Boyd, G. P. Agrawal, and P. M. Fauchet, "Optical solitons in a silicon waveguide," Opt. Express, vol. 15, no. 12, pp. 7682-7688, Jun. 2007.

[7] H. Ji, M. Galili, H. Hu, M. Pu, L. K. Oxenløwe, K. Yvind, J. M. Hvam, and P. Jeppesen, "1.28-Tb/s demultiplexing of an OTDM DPSK data signal using a silicon waveguide," IEEE Photon. Technol. Lett., vol. 22, no. 23, pp. 1762-1764, Dec. 2010.

[8] D. C. Zografopoulos, R. Beccherelli, and E. E. Kriezis, "Quasi-soliton propagation in dispersion-engineered silicon nanowires," Opt. Commun., vol. 285, no. 15, pp. 3306-3311, Jul. 2012.

[9] B. A. Daniel and G. P. Agrawal, "Vectorial nonlinear propagation in silicon nanowire waveguides: polarization effects," J. Opt. Soc. Am. B, vol. 27, no. 5, pp. 956-965, May 2010.

[10] A. C. Turner-Foster, M. A. Foster, J. S. Levy, C. B. Poitras, R. Salem, A. L. Gaeta, and M. Lipson, "Ultrashort free-carrier lifetime in low-loss silicon nanowaveguides," Opt. Express, vol. 18, no. 4, pp. 3582-3591, Feb. 2010.

[11] L. Yin, Q. Lin, and G. P. Agrawal, "Soliton fission and supercontinuum generation in silicon waveguides," Opt. Lett., vol. 32, no. 4, pp. 391-393, Feb. 2007.

[12] B. Tatian, "Fitting refractive-index data with the Sellmeier dispersion formula," Appl. Opt., vol. 23, no. 24, pp. 4477-4485, Dec. 1984.

[13] L. Yin and G. P. Agrawal, "Impact of two-photon absorption on selfphase modulation in silicon waveguides," Opt. Lett., vol. 32, no. 14, pp. 2031-2033, Jul. 2007.

[14] N. C. Panoiu, X. Liu, and R. M. Osgood, Jr., "Self-steepening of ultrashort pulses in silicon photonic nanowires," Opt. Lett., vol. 34, no. 7, pp. 947-949, Apr. 2009.

[15] X. Chen, N. C. Panoiu, and R. M. Osgood, Jr., "Theory of Ramanmediated pulsed amplification in silicon-wire waveguides," IEEE $J$. Quantum Electron., vol. 42, no. 2, pp. 160-170, Feb. 2006.

[16] S. Afshar V. and T. M. Monro, "A full vectorial model for pulse propagation in emerging waveguides with subwavelength structures part I: Kerr nonlinearity," Opt. Express, vol. 17, no. 4, pp. 2298-2318, Feb. 2009.

[17] G. P. Agrawal, Nonlinear Fiber Optics, 4th ed. Academic Press, 2007.

[18] J. B. Driscoll, N. Ophir, R. R. Grote, J. I. Dadap, N. C. Panoiu, K. Bergman, and R. M. Osgood, Jr., "Width-modulation of Si photonic wires for quasi-phase-matching of four-wave-mixing: experimental and theoretical demonstration," Opt. Express, vol. 20, no. 8, pp. 9227-9242, Apr. 2012.

[19] H. Yoda, K. Shiraishi, A. Ohshima, T. Ishimura, H. Furuhashi, H. Tsuchiya, and C. Tsai, "A two-port single-mode fiber-silicon wire waveguide coupler module using spot-size converters," J. Lightw. Technol., vol. 27, no. 10, pp. 1315-1319, May 2009.

[20] B. Lee, X. Chen, A. Biberman, X. Liu, I. Hsieh, C.-Y. Chou, J. Dadap, F. Xia, W. Green, L. Sekaric, Y. Vlasov, R. M. Osgood, Jr., and $\mathrm{K}$. Bergman, "Ultrahigh-bandwidth silicon photonic nanowire waveguides for on-chip networks," IEEE Photon. Technol. Lett., vol. 20, no. 6, pp. 398-400, Mar. 2008. 